\title{
THE INFLUENCE OF ANTERIOR ESTHETIC FIXED APPLIANCE OF PREMATURELY LOST PRIMARY INCISORS ON SOUND PRODUCTION IN A GROUP OF EGYPTIAN CHILDREN. (A CASE CONTROL STUDY)
}

\author{
Adel Abdel Azeem Elbardissy", Sara A. Mahmoud* and Asmaa Abdel Hamid ${ }^{* *}$
}

\begin{abstract}
Background: premature loss of primary incisors could affect speech, esthetics, and arch integrity. The need for replacement of prematurely lost primary incisors with esthetic and functional fixed or removable appliances were a subject for investigation in many literatures. The aim of such appliances is to restore esthetics, preserve arch integrity and to prevent speech impairment.
\end{abstract}

Aim of the study: to evaluate the Influence of anterior esthetic fixed appliance of prematurely lost primary incisors on sound production and speech intelligibility.

Methodology: A case control study was conducted on 15 children (3-5 years old) with premature loos of primary incisors (experimental group) and 15 children of similar age completely dentulous. Speech evaluation was examined in four stages: Before extraction of the decayed primary incisors. Before the modified fixed partial denture fitting. Immediately after denture fitting. One month after denture fitting.

Results: sound production and speech intelligibility were not affected by premature loss of primary teeth. Minor affection after immediate placement of the fixed prosthetic appliance was restored in a period of one month as a result of adaptation.

Conclusions: 1- Early loss of primary incisors due to caries would have minor effect on speech production 2- Replacement of prematurely lost primary incisors with fixed prosthetic appliances did not affect speech production 3- Replacement of prematurely lost primary incisors with fixed prosthetic appliances for the purpose of restoring appearance if required by the parents should be encouraged however, assuring that speech development will not be affected by early loss of these teeth should also be explained to them if they are concerned.

KEY WORDS: premature loss of primary incisors, speech intelligibility, speech production

* Associate Professor of Pediatric Dentistry- Faculty of Dentistry - Cairo University

** Associate Professor of phoniatrics, phoniatric unit. ENT department, Faculty of Medicine, Cairo University. 


\section{INTRODUCTION}

Premature loss of primary anterior teeth due to trauma or extensive caries with poor prognosis occurs frequently in young children ${ }^{(1)}$. It can affect esthetics, eating, speech development, arch integrity, development and eruption of the permanent successors, quality of life, and could results in development of oral habits ${ }^{(2)}$.

Relationship between the dentition and speech production, especially for the anterior teeth has been documented in many literatures ${ }^{(3-7)}$.

Speech development and the ability to articulate certain speech sounds are dependent on many related factors among which is the presence of the anterior maxillary teeth ${ }^{(8)}$.

Sudden premature loss of primary incisors due to trauma could result in a more degree of speech impairment than extracted carious teeth that are no longer restorable. Unlike the sudden loss of primary incisors due to trauma, destruction of the crowns because of early childhood caries is relatively slow and allows adaptation of articulation to the gradual changing condition ${ }^{(1)}$.

Speech impairment due to premature loss of primary incisors is more obvious in children younger than three years of age ${ }^{(8,9)}$. Premature loss of primary incisors could lead to anterior dental disharmonies which will interfere with the normal tongue placement which then can lead to the development of maladaptive articulatory habits. Tongue thrust commonly develops when missing anterior teeth are not replaced ${ }^{(10)}$. Yet another consideration is the child's speech development following missing incisors. Many sounds are made with the tongue touching the lingual side of the maxillary incisors, and inappropriate speech compensations can happen ${ }^{(11)}$.

One study demonstrated that children who had worn dentures from early childhood exhibited no articulation errors, while those who did not, exhibited articulation errors directly related to dentition. This study concluded that patients who received prosthetic dental appliances develop better articulation skills ${ }^{(12)}$.

There are many types of appliances that can be fabricated for replacement of prematurely lost primary incisors ${ }^{(13-16) .}$ Removable partial dentures or modified fixed ones were suggested. Fixed appliances proved to be more practical and long lasting. Groper appliance ${ }^{(17)}$ is a fixed appliance similar to a Nance holding arch, but with plastic teeth processed onto the wire instead of a palatal acrylic button in the rugae area. The round wire should be 0.036 to 0.040 inch in diameter and is attached to either the first or second primary molars with either stainless steel crowns (SSC) or prefabricated stainless steel bands. The plastic or acrylic teeth are attached to metal cleats that have been soldered to the palatal wire bar. The teeth sit directly on the alveolar crest without any gingival colored acrylic extending into the vestibule or onto the palate.

This study was done to evaluate the influence of modified Groper appliances on sound production in a group of Egyptian children with prematurely lost primary incisors.

\section{SUBJECTS AND METHODS:}

This study was designed and carried out in the Department of Pediatric Dentistry and dental Public Health,

The study sample included thirty children in age ranged from 3-5 years old.

The mother tongue of all of the subjects was Arabic, and they had no medical problems and had normal hearing.

The present study was planned as a case-control study. Selection criteria of the case group (15 children) included at least one primary maxillary incisor indicated for extraction. 
The control group (15 children) was completely dentulous subjects with healthy primary incisors and normal speech pattern as regard his/her age.

The aim and clinical procedure of the study were explained and an informed consent was obtained from the children's parents.

The case group was subjected to the following protocol:

- Case history included personal, past and present medical history, as well as, dental history.

- Clinical examination and treatment planning for the expected early loss upper anterior primary incisors.

- Extraction of badly decayed primary incisors.

- Prosthetic treatment by placing a modified fixed partial denture.

Steps of appliance fabrication:

First appointment:

1. The appropriate band for the primary molars were selected, tried and adjusted.

2. If the selected molars were in need for pulpotomies bands were replaced by stainless steel crowns.

3. Alginate dental impressions (Tropicalgin) (Zhermack GmbH, Germany) were taken with the bands or the SSCs on teeth. Then they were poured with dental stone with the bands or the SSCs in the impression.

4. Appliances were manufactured in the dental lab according to Groper design ${ }^{(20)}$ using an acrylic plate for teeth attachment as a modification.

Second appointment:

1. Appliances were tried, adjusted as needed and cemented with glass ionomer cement

(PROMEDICA Dental Material GmbH, Germany) (Fig. 1).

\section{Speech evaluation:}

Speech evaluation was done by a Phoniatrician who is experienced in the speech therapy of preschool children. All children were subjected to the protocol of speech evaluation and assessment applied in Phoniatric unit, Cairo University:

I Articulation test: to exclude preexisting phonological errors ${ }^{(18)}$.

\section{II- Auditory perceptual assessment for speech intelligibility:}

Three speech sounds were chosen to be studied in the present study/s/ /z/ / / /:

A sample of ten sentences each consists of average ten words for each phoneme under study.

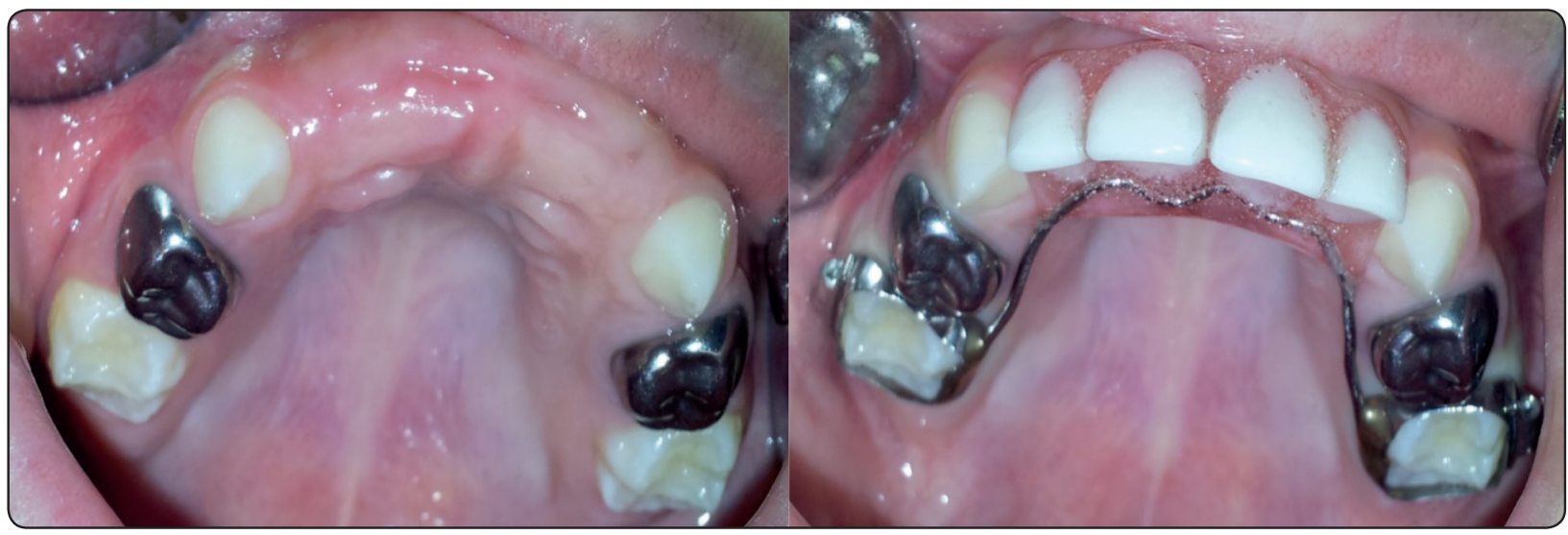

Fig (1) Adjustment and fixation of the Groper appliance. 
A sentence involving the phoneme in various word positions (Initial, Middle, Terminal) was introduced.

The children were asked to repeat the sentences. The samples were digitally recorded \& thereafter subjectively analyzed by three un-familiar nonprofessionals.

- Each listener was asked to give a score-out of ten for each introduced sentence according to the number of intelligible (clear or understood word).

- The score obtained by the three listeners for each sentence were summed $\&$ divided by three to obtain the average intelligibility score for each studied sound.

- The mean intelligibility scores were calculated, tabulated \& prepared for statistical analysis.

The general intelligibility score was calculated using: Speech intelligibility in context:

\section{5 -point scale:}

Grade 1: Speech is completely un-intelligible.

Grade 2: Speech is very difficult to be understood; only isolated words or phrases are intelligible.

Grade 3: The listener can understand with difficulty about half the content of the message.

Grade 4: Speech is intelligible with exception of a few words or phrases.

Grade 5: Speech is completely intelligible.

General intelligibility score was calculated by taking the mean of three non-professional listeners scoring results.

\section{III) Spectrographic analysis:}

Using the computerized speech lab (CSL 4300 B), the child was seated in an upright position \& allowed to talk freely \& repeat syllables said to him / her. The microphone was fixed about twenty $\mathrm{cm}$ away from the child's mouth.

\section{Each child was evaluated at four sittings:}

Initial sample: Before extraction of the decayed primary incisors.

Second Sample: Before the modified fixed partial denture insertion (Pre-fitting).

Third Sample: Immediately after the denture fitting.

Fourth sample: One month after denture fitting.

The same protocol of assessment was utilized for the control group (normal subjects); however recordings were made for the control group only once.

The speech Samples: Six Arabic consonants placed with the vowel in vowel-consonant- vowel (V-C-V) syllabic form e.g. / æ s æ /, / æ $\int \mathfrak{x} /, / \mathfrak{z}$ $æ /$. Thereafter, spectrographic analysis was made. The following parameters were obtained after that.

1) Consonant durations: in mseconds (ms).

2) Consonant energy: in decibel (dB)

\section{RESULTS}

\section{Statistical analysis plan}

\section{Descriptive analyses:}

Each variable is described in terms of frequencies and percentages (Number of missing teeth, intelligibility scores and errors of speech sounds) or mean, median, standard deviation (SD) and range (age, speech sounds energy and duration).

a. Test for normality: To test the normality of the continuous data, the Shapiro-Wilk test for normality was applied.

\section{b. Comparative analysis:}

(a) Speech sounds energy and duration: Normally distributed data required using the parametric Paired Student's t-test to assess the differences across different follow ups in the test group. Unpaired t-test was applied to assess the 
differences between test and control group. Data that was not normally distributed required using the non-parametric Mann-Whitney U test.

(b) Intelligibility scores and errors of speech sounds of the test group: Paired Wilcoxon Singed Ran Sum test was used.

\section{c. The significance level:}

It was verified at $P \leq 0.05$. The results are considered to be statistically significant if $\mathrm{p}$-value was less than 0.05 .

\section{d. Statistical package used for this study*: $\mathrm{R}$} statistical package, version 2.15.2 (26-10-2012) was used for analyzing the data. Copyright (C) 2012 - The R Foundation for Statistical Computing.

TABLE (1): Descriptive analysis of number of missing teeth of the test group- Frequency (Number of cases) and percentage. $(n=15)$

\begin{tabular}{|l|l|l|}
\hline & Frequency & Percentage \\
\hline One tooth & 3 & $20 \%$ \\
\hline Two teeth & 5 & $33.33 \%$ \\
\hline Three teeth & 1 & $6.67 \%$ \\
\hline Four teeth & 6 & $40 \%$ \\
\hline
\end{tabular}

- As shown in table (1), the highest proportion (40\%) of the patients have four missing teeth, followed by those who have two missing teeth $(33.33 \%)$, then those who have one missing tooth $(20 \%)$. The lowest percentage belongs to those who have three missing teeth $(6.67 \%)$.

TABLE (2): Descriptive analysis of age (months) of the test group- Mean, median, standard deviation (SD) and range. $(\mathrm{n}=15)$

\begin{tabular}{|l|c|c|c|c|c|}
\hline & \multirow{2}{*}{ Mean } & \multirow{2}{*}{ Median } & \multirow{2}{*}{ SD } & \multicolumn{2}{|c|}{ Range } \\
\cline { 5 - 6 } & & & & Min & Max \\
\hline Age (months) & 50 & 48 & 7.63 & 36 & 60 \\
\hline
\end{tabular}

- As shown in table (2) the mean age of the test group patients is 50 months $( \pm 7.63$ months) which equals to 4.17 years.

TABLE (3): Descriptive analysis of intelligibility scores of the test group across different follow ups - Frequency (Number of cases) and percentage. $(\mathrm{N}=15)$

\begin{tabular}{|c|c|c|c|}
\hline $\begin{array}{c}\text { Follow-up } \\
\text { Grade }\end{array}$ & Pre-fitting & Immediately & After one month \\
\hline Grade 1 & $0(0 \%)$ & $2(13.33 \%)$ & $0(0 \%)$ \\
\hline Grade 2 & $5(33.33 \%)$ & $4(26.67 \%)$ & $0(0 \%)$ \\
\hline Grade 3 & $4(26.67 \%)$ & $6(40 \%)$ & $1(6.67 \%)$ \\
\hline Grade 4 & $5(33.33 \%)$ & $2(13.33 \%)$ & $7(46.67 \%)$ \\
\hline Grade 5 & $1(6.67 \%)$ & $1(6.67 \%)$ & $7(46.67 \%)$ \\
\hline
\end{tabular}

TABLE (4): Descriptive analysis of Speech errors of "S" sound of the test group across different follow ups - Frequency (Number of cases) and percentage. $(\mathrm{N}=15)$

\begin{tabular}{|c|c|c|c|}
\hline $\begin{array}{c}\text { "S" Sound } \\
\text { Speech } \\
\text { Errors }\end{array}$ & $\begin{array}{c}\text { After } \\
\text { extraction }\end{array}$ & $\begin{array}{c}1 \text { week after } \\
\text { denture } \\
\text { fitting }\end{array}$ & $\begin{array}{c}1 \text { month } \\
\text { after denture } \\
\text { fitting }\end{array}$ \\
\hline Normal & $\begin{array}{c}10 \\
(66.67 \%)\end{array}$ & $\begin{array}{c}12 \\
(80 \%)\end{array}$ & $\begin{array}{c}13 \\
(86.67 \%)\end{array}$ \\
\hline $\begin{array}{c}\text { Interdental } \\
\text { lisping }\end{array}$ & $\begin{array}{c}5 \\
(33.33 \%)\end{array}$ & 3 & 2 \\
$(20 \%)$ & $(13.33 \%)$ \\
\hline
\end{tabular}

TABLE (5): Descriptive analysis of Speech errors of " $Z$ " sound of the test group across different follow ups - Frequency (Number of cases) and percentage. $(\mathrm{N}=15)$

\begin{tabular}{|c|c|c|c|}
\hline $\begin{array}{c}\text { "Z" Sound } \\
\text { Erroech }\end{array}$ & $\begin{array}{c}\text { After } \\
\text { extraction }\end{array}$ & $\begin{array}{c}1 \text { week after } \\
\text { denture } \\
\text { fitting }\end{array}$ & $\begin{array}{c}1 \text { month } \\
\text { after denture } \\
\text { fitting }\end{array}$ \\
\hline Normal & $\begin{array}{c}9 \\
(60 \%)\end{array}$ & $\begin{array}{c}9 \\
(60 \%)\end{array}$ & $\begin{array}{c}12 \\
(80 \%)\end{array}$ \\
\hline Substitution & $\begin{array}{c}1 \\
(6.67 \%)\end{array}$ & $\begin{array}{c}1 \\
(6.67 \%)\end{array}$ & $\begin{array}{c}1 \\
(6.67 \%)\end{array}$ \\
\hline $\begin{array}{c}\text { Interdental } \\
\text { lisping }\end{array}$ & 5 & 5 & 2 \\
$(33.33 \%)$ & $(33.33 \%)$ & $(13.33 \%)$ \\
\hline
\end{tabular}


TABLE (6): Descriptive analysis of Speech errors of / $\int /$ sound of the test group across different follow ups - Frequency (Number of cases) and percentage. $(\mathrm{N}=15)$

\begin{tabular}{|c|c|c|c|}
\hline "SH" Sound Speech Errors & After extraction & 1 week after denture fitting & 1 month after denture fitting \\
\hline Normal & $14(93.33 \%)$ & $14(93.33 \%)$ & $14(93.33 \%)$ \\
\hline Substitution & $1(6.67 \%)$ & $0(0 \%)$ & $0(0 \%)$ \\
\hline Distortion & $0(0 \%)$ & $1(6.67 \%)$ & $1(6.67 \%)$ \\
\hline
\end{tabular}

TABLE (7): Comparison between test and control group regarding /s/ Sound Energy - Mean difference (MD), standard deviation (SD) and results of the Student's t-test (p-value).

\begin{tabular}{|c|c|c|c|c|c|}
\hline \multirow{2}{*}{\multicolumn{2}{|c|}{ /s/ Sound Energy $(\mathrm{dB})$}} & \multirow{2}{*}{ MD } & \multirow{2}{*}{$\begin{array}{c}\text { SD } \\
\text { p-value* }\end{array}$} & Student's t-test & \\
\hline & & & & Interpretation & \\
\hline Pre Prosthesis & \multirow{3}{*}{ Pre Extraction } & -0.62 & 0.96 & 0.0251 & Statistically significant difference \\
\hline Immediate & & 1.4 & 4.18 & $0.1321 * *$ & No difference \\
\hline After One Month & & 3.7 & 3 & 0.00029 & Statistically significant difference \\
\hline Immediate & \multirow{2}{*}{ Pre Prosthesis } & 2.02 & 4.31 & $0.1182 * *$ & No difference \\
\hline After One Month & & 4.33 & 3.16 & 0.00011 & Statistically significant difference \\
\hline After One Month & Immediate & 2.31 & 3.93 & $0.02481 * *$ & Statistically significant difference \\
\hline \multirow{4}{*}{ Control } & Pre Extraction & 6.63 & 4.83 & 0.0013 & Statistically significant difference \\
\hline & Pre Prosthesis & 7.26 & 4.93 & 0.00043 & Statistically significant difference \\
\hline & Immediate & 5.24 & 6.05 & $0.06793 * *$ & Statistically significant difference \\
\hline & After One Month & 2.93 & 5.19 & 0.1507 & No difference \\
\hline
\end{tabular}

*Statistical significance at p-value $\leq \mathbf{0 . 0 5}$.

**Results of the non-parametric Mann-Whitney $U$ test.

TABLE (8): Comparison between test and control group regarding /s/ Sound Duration - Mean difference (MD), standard deviation (SD) and results of the Student's t-test (p-value).

\begin{tabular}{|c|c|c|c|c|c|}
\hline \multirow{2}{*}{\multicolumn{2}{|c|}{ /s/ Sound duration (ms) }} & \multirow{3}{*}{$\begin{array}{l}\text { MD } \\
0.01\end{array}$} & \multirow{3}{*}{\begin{tabular}{|l|} 
SD \\
0.02 \\
\end{tabular}} & \multicolumn{2}{|c|}{ Student's t-test } \\
\hline & & & & p-value* & Interpretation \\
\hline Pre Prosthesis & \multirow{3}{*}{ Pre Extraction } & & & 0.1843 & No difference \\
\hline Immediate & & 0.03 & 0.05 & 0.0577 & No difference \\
\hline After One Month & & 0.02 & 0.05 & 0.1711 & No difference \\
\hline Immediate & \multirow{2}{*}{ Pre Prosthesis } & 0.02 & 0.05 & 0.1563 & No difference \\
\hline After One Month & & 0.01 & 0.05 & 0.3059 & No difference \\
\hline After One Month & Immediate & -0.01 & 0.05 & 0.5688 & No difference \\
\hline \multirow{4}{*}{ Control } & Pre Extraction & 0 & 0.06 & $0.9338 * *$ & No difference \\
\hline & Pre Prosthesis & -0.01 & 0.06 & $0.8682 * *$ & No difference \\
\hline & Immediate & -0.03 & 0.06 & $0.2286 * *$ & No difference \\
\hline & After One Month & -0.02 & 0.06 & $0.4799 * *$ & No difference \\
\hline
\end{tabular}


TABLE (9): Comparison between test and control group regarding /z/ Sound Energy - Mean difference (MD), standard deviation (SD) and results of the Student's t-test (p-value).

\begin{tabular}{|c|c|c|c|c|c|}
\hline \multirow{2}{*}{\multicolumn{2}{|c|}{ /z/ Sound Energy $(\mathrm{dB})$}} & \multirow{3}{*}{$\begin{array}{c}\text { MD } \\
-1.84\end{array}$} & \multirow{3}{*}{$\begin{array}{c}\mathrm{SD} \\
1.41\end{array}$} & \multicolumn{2}{|r|}{ Student's t-test } \\
\hline & & & & p-value* & Interpretation \\
\hline Pre Prosthesis & \multirow{3}{*}{ Pre Extraction } & & & $0.00085^{* *}$ & Statistically significant difference \\
\hline Immediate & & 2.27 & 3.20 & 0.01574 & Statistically significant difference \\
\hline After One Month & & 6.95 & 4.43 & $<0.00001$ & Statistically significant difference \\
\hline Immediate & \multirow{2}{*}{ Pre Prosthesis } & 4.11 & 2.57 & $0.00072 * *$ & Statistically significant difference \\
\hline After One Month & & 8.80 & 4.02 & $0.00072 * *$ & Statistically significant difference \\
\hline After One Month & Immediate & 4.69 & 2.66 & $<0.00001$ & Statistically significant difference \\
\hline \multirow{4}{*}{ Control } & Pre Extraction & 6.78 & 6.35 & 0.00613 & Statistically significant difference \\
\hline & Pre Prosthesis & 8.63 & 5.80 & $0.0003 * *$ & Statistically significant difference \\
\hline & Immediate & 4.52 & 4.49 & 0.03675 & Statistically significant difference \\
\hline & After One Month & -0.17 & 2.96 & 0.9249 & No difference \\
\hline
\end{tabular}

*Statistical significance at p-value $\leq 0.05$.

**Results of the non-parametric Mann-Whitney $U$ test.

TABLE (10): Comparison between test and control group regarding /z/ Sound Duration - Mean difference (MD), standard deviation (SD) and results of the Mann-Whitney U test (p-value).

\begin{tabular}{|c|c|c|c|c|c|}
\hline \multirow{2}{*}{\multicolumn{2}{|c|}{ /z/ Sound duration (ms) }} & \multirow{3}{*}{$\begin{array}{l}\text { MD } \\
0.03\end{array}$} & \multirow{3}{*}{$\begin{array}{c}\text { SD } \\
0.05\end{array}$} & \multicolumn{2}{|r|}{ Mann-Whitney U test } \\
\hline & & & & p-value* & Interpretation \\
\hline Pre Prosthesis & \multirow{3}{*}{ Pre Extraction } & & & 0.02304 & Statistically significant difference \\
\hline Immediate & & 0.01 & 0.04 & 0.7615 & No difference \\
\hline After One Month & & 0 & 0.04 & 0.7982 & No difference \\
\hline Immediate & \multirow{2}{*}{ Pre Prosthesis } & -0.02 & 0.04 & 0.03809 & Statistically significant difference \\
\hline After One Month & & -0.03 & 0.05 & 0.04791 & Statistically significant difference \\
\hline After One Month & Immediate & -0.01 & 0.03 & 0.01706 & Statistically significant difference \\
\hline \multirow{4}{*}{ Control } & Pre Extraction & 0.02 & 0.07 & 0.7397 & No difference \\
\hline & Pre Prosthesis & -0.01 & 0.07 & 0.4803 & No difference \\
\hline & Immediate & 0.01 & 0.05 & 0.5896 & No difference \\
\hline & After One Month & 0.02 & 0.05 & 0.07404 & No difference \\
\hline
\end{tabular}

*Statistical significance at p-value $\leq \mathbf{0 . 0 5}$. 
TABLE (11): Comparison between test and control group regarding / $\int /$ Sound Energy - Mean difference (MD), standard deviation (SD) and results of the Mann-Whitney U test (p-value).

\begin{tabular}{|c|c|c|c|c|c|}
\hline \multirow{2}{*}{\multicolumn{2}{|c|}{$/$ / / Sound Energy $(\mathrm{dB})$}} & \multirow{3}{*}{\begin{tabular}{|l|} 
MD \\
-1.13
\end{tabular}} & \multirow{3}{*}{\begin{tabular}{|l|} 
SD \\
1.54
\end{tabular}} & \multicolumn{2}{|c|}{ Mann-Whitney U test } \\
\hline & & & & p-value* & Interpretation \\
\hline Pre Prosthesis & \multirow{3}{*}{ Pre Extraction } & & & $0.01317 * *$ & Statistically significant difference \\
\hline Immediate & & 1.68 & 6.88 & 0.2678 & No difference \\
\hline After One Month & & 3.87 & 6.20 & $0.02991 * *$ & Statistically significant difference \\
\hline Immediate & \multirow{2}{*}{ Pre Prosthesis } & 2.81 & 6.55 & 0.09364 & No difference \\
\hline After One Month & & 5 & 6.18 & $0.007364 * *$ & Statistically significant difference \\
\hline After One Month & Immediate & 2.18 & 5.38 & 0.1817 & No difference \\
\hline \multirow{4}{*}{ Control } & Pre Extraction & -0.49 & 7.59 & 1 & No difference \\
\hline & Pre Prosthesis & 0.65 & 7.95 & 0.9338 & No difference \\
\hline & Immediate & -2.17 & 7.51 & 0.3833 & No difference \\
\hline & After One Month & -4.35 & 7.96 & 0.02496 & Statistically significant difference \\
\hline
\end{tabular}

$*$ Statistical significance at $\mathrm{p}$-value $\leq 0.05 . * *$ Results of the parametric paired $\mathrm{t}$ - test.

TABLE (12): Comparison between test and control group regarding / / Sound Duration - Mean difference (MD), standard deviation (SD) and results of the Mann-Whitney U test (p-value).

\begin{tabular}{|c|c|c|c|c|c|}
\hline \multirow{2}{*}{\multicolumn{2}{|c|}{ / J/ Sound Duration (ms) }} & \multirow{3}{*}{\begin{tabular}{|l|} 
MD \\
0
\end{tabular}} & \multirow{3}{*}{$\begin{array}{l}\text { SD } \\
0.03\end{array}$} & \multicolumn{2}{|c|}{ Mann-Whitney U test } \\
\hline & & & & n-value* & Internretation \\
\hline Pre Prosthesis & \multirow{3}{*}{ Pre Extraction } & & & $0.7412 * *$ & No difference \\
\hline Immediate & & 0.01 & 0.06 & 0.7982 & No difference \\
\hline After One Month & & -0.02 & 0.06 & $0.227 * *$ & No difference \\
\hline Immediate & \multirow{2}{*}{ Pre Prosthesis } & 0.01 & 0.06 & 0.6829 & No difference \\
\hline After One Month & & -0.02 & 0.06 & $0.1998 * *$ & No difference \\
\hline After One Month & Immediate & -0.03 & 0.06 & 0.04978 & No difference \\
\hline \multirow{4}{*}{ Control } & Pre Extraction & -0.02 & 0.05 & 0.08845 & No difference \\
\hline & Pre Prosthesis & -0.02 & 0.05 & 0.07071 & No difference \\
\hline & Immediate & -0.03 & 0.07 & 0.05606 & No difference \\
\hline & After One Month & 0 & 0.04 & 0.6623 & No difference \\
\hline
\end{tabular}

$*$ Statistical significance at p-value $\leq \mathbf{0 . 0 5}$.

**Results of the parametric paired t- test. 
TABLE (13): Comparison between intelligibility scores of test group across different follow ups- Results of the Wilcoxon Signed Rank Sum test (p-value).

\begin{tabular}{|c|c|c|c|}
\hline \multicolumn{2}{|c|}{ Intelligibility Scores } & \multicolumn{2}{|c|}{ Wilcoxon Signed Rank Sum test* } \\
\hline & & p-value $* *$ & Interpretation \\
\hline Immediate & \multirow{2}{*}{ Pre-fitting } & 0.04108 & Statistically significant difference \\
\hline After One Month & & 0.0007167 & Statistically significant difference \\
\hline After One Month & Immediate & 0.0008744 & Statistically significant difference \\
\hline
\end{tabular}

*Wilcoxon Signed Rank Sum test for paired data

*Statistical significance at p-value $\leq 0.05$.

TABLE (14): Comparison between /s/ Sound Speech Errors of test group across different follow upsResults of the Wilcoxon Signed Rank Sum test (p-value).

\begin{tabular}{|l|l|l|l|}
\hline \multicolumn{2}{|l|}{ /s/ Sound Speech Errors } & \multicolumn{2}{l|}{ Wilcoxon Signed Rank Sum test* } \\
\cline { 3 - 4 } & \multirow{2}{*}{ After extraction } & p-value** & Interpretation \\
\hline 1 week after denture fitting & & 0.5 & No difference \\
\hline 1 month after denture fitting & $\mathbf{1}$ week after denture fitting & 0.5 & No difference \\
\hline 1 month after denture fitting & & 0.5 & No difference \\
\hline
\end{tabular}

*Wilcoxon Signed Rank Sum test for paired data $\quad *$ Statistical significance at p-value $\leq 0.05$.

TABLE (15): Comparison between /z/ Sound Speech Errors of test group across different follow ups- Results of the Wilcoxon Signed Rank Sum test (p-value).

\begin{tabular}{|l|l|l|l|}
\hline \multicolumn{2}{|l|}{ /z/ Sound Speech Errors } & \multicolumn{2}{l|}{ Paired Wilcoxon Signed Rank Sum test } \\
\cline { 3 - 4 } & \multirow{2}{*}{ After extraction } & p-value** & Interpretation \\
\hline 1 week after denture fitting & & 0.3458 & No difference \\
\cline { 3 - 4 } 1 month after denture fitting & & 0.5 & No difference \\
\hline 1 month after denture fitting & 1 week after denture fitting & 0.5 & No difference \\
\hline
\end{tabular}

*Wilcoxon Signed Rank Sum test for paired data

*Statistical significance at p-value $\leq \mathbf{0 . 0 5}$.

Table (16): Comparison between / $\int /$ Sound Speech Errors of test group across different follow ups- Results of the Wilcoxon Signed Rank Sum test (p-value).

\begin{tabular}{|l|l|l|l|}
\hline \multirow{2}{*}{$/ \int /$ sound errors } & \multicolumn{2}{l|}{ Paired Wilcoxon Signed Rank Sum test } \\
\cline { 3 - 4 } & \multirow{2}{*}{ After extraction } & p-value** & Interpretation \\
\hline 1 week after denture fitting & & 0.3458 & No difference \\
\cline { 3 - 4 } 1 month after denture fitting & $\mathbf{1}$ week after denture fitting & 0.3458 & No difference \\
\hline 1 month after denture fitting & & 0.5 & No difference \\
\hline
\end{tabular}




\section{DISCUSSION}

The specific articulatory processes that result in human speech have intrigued researchers for many years. Speech is communicated from speaker to listener primarily through sound waves. This acoustic speech signal is created through a combination of complex steps that result in the signal we perceive as meaningful communication. These steps include the movement of the articulators to help shape the acoustic realization of different speech sounds ${ }^{(19)}$.

Dental prosthesis is a foreign body in oral cavity and thus necessarily interferes with speech articulation ${ }^{(19)}$. The purpose of this study was to evaluate the Influence of anterior esthetic fixed appliance of prematurely lost primary incisors on sound production and speech intelligibility

Selected 3 speech sounds are: Palatal sound / / \& Linguoalveolar sounds /s/, /z/. These sounds were selected as fricatives that need spreading of air through the anterior teeth (incisors) in their sound production and this may be affected by the prematurely lost primary incisors or their replacement by fixed appliances applied in this study. Speech was evaluated through objective \& subjective methods.

\section{As regard sound /s/}

Comparison of cases and controls as regard energy (table7) revealed high significant difference at the 3 setting (pre extraction, pre-prosthesis and immediately after fitting) and non-significant difference after 1 month of fitting. That means increased sound energy after adaptation to the prosthesis. this is accompanied by increased general intelligibility score after one month to grade 4 and 5 in $46.67 \%$ of cases as shown in (table 3) with less improvement of intelligibility immediately after fitting (that explained by adaptation to denture).

Also high significant difference was found between pre extraction and both pre prosthesis and after one month but no difference with immediate after extraction. High significant difference also was found between immediate and after 1 month of extraction revealed the effect of adaptation on increased sound energy.

As regard duration of sound /s/, all sound durations show non-significant difference between controls and all follow ups of the test groups and this may indicate that the duration is not the only a mean contributing factor in intelligibility of sound $/ \mathrm{s} /$. This can be explained by the nature of mechanism of production of sound /s/ (linguoalveolar sound) in which a groove is often formed along the tongue midline to channel the air stream. This is accomplished by touching the sides of the tongue to the sides of the teeth ${ }^{(20)}$.

Speech errors including /s/ sound as shown in (table 4) revealed $10(66.67 \%)$ cases with normal /s/ production and 5 cases (33.33\%) with interdental lipsing after extraction , that decreased interdental lipsing after 1 week and after 1 month(20\%) of denture fitting (13.33\%). This also may explain improvement of intelligibility after 1 week and after 1 month of extraction.

\section{As regards /z/ sound}

It is closely similar to /s/ sounds as regard place and manner of articulation but /z/sound is a voiced sound that requires vocal fold vibration for its production that is not affected in the test. Nonsignificant difference between cases and controls in the energy of sound /z/ was found after 1 month of fitting that revealed improvement as in sound /s/. Non-significant difference in duration between cases and controls was also found, speech errors were also improved after 1 month (table 5 )

\section{As regard sound $/ \int /$}

As regard energy of sound $/ \int /$ (table 12) revealed higher sound energy after fitting than that of pre-prosthesis energy that indicate improvement of pronunciation of the sounds, non-significant difference in energy was also found between immediate and after 1 month of fitting, this may be 
explained by rapid improvement of sound / / after fitting

$/ \int /$ is a palatal sound depends on its production of spreading of air. this coincides with finding of Molly et al., $2008^{(21)}$ who found that linguoalveolar fricatives (such as /s/and / $/$ / ) are more sensitive in their pronunciation than other fricatives, as they require adequate spreading of the air through the teeth, high precision movement of the tongue as well as controlled jaw positioning.

Non-significant difference in / $/$ / energy between controls and all follow ups of test group (which revealed improvement of $/ \int /$ sound pronunciation) except 1 month after fitting is statistically higher than that of control group, which may be explained by intended correction by the child with over pronunciation of the sound and that is approved with analysis sound error in which $/ \int /$ sound is not affected from the start (after extraction)except in 1 case $(6.6 \%)$ and distortion in 1 case $(6.6 \%)$ after 1 week and 1 month of fitting

Better results were also observed after this duration (after one months), this could be explained by adaptation of the tongue. Due to its flexibility, the tongue is directly involved in the production of the majority of human speech sounds ${ }^{(22)}$. It is acknowledged by Niemi et al. ${ }^{(23)}$ that the tongue plays the greatest of all roles in that adaptive process

The result of this study showed that speech affection as a result of premature loss of primary incisors at age between 3-5 years and due to dental caries was not significant. These results are in agreement with the results of Gable et al ${ }^{(24)}$. This was explained by Riekman and ElBadrawy ${ }^{(8)}$ who reported that 4 of 14 children who prematurely lost maxillary primary incisors due to early childhood caries had some degree of speech impairment with 2 being severe. All those with speech impairment had the extractions prior to age 3 , while those with extractions at a later age were less likely to do so. Moreover, it can be concluded that when primary incisors reach a stage that they are no longer restorable; their crowns may have lost much of their original form and thus their role in articulation on the contrary to sudden loss of primary incisors due to trauma. Loss of crown structure in early childhood caries is relatively slow and might allow adaptation of articulation to the gradual changing condition, which may explain why there were less speech problems when teeth were extracted after the age of three.

Fitting a prosthetic replacement for prematurely extracted primary incisors had some impairment on speech after immediate placement of the appliances. Improvement of speech after one month was significant and was explained by adaptation of the articulating structure with the new fitted appliance.

The most important reason for replacing missing incisors is to restore a natural and pleasing appearance ${ }^{(18)}$. This will provide an opportunity for normal psychological development. Children who regularly attend daycare or preschool programs may become more aware of their image and lack of teeth and be affected by their appearance. As these children approach school age, they may have a lesser problem fitting into groups of children who are in the mixed dentition and actively exfoliating the primary incisors ${ }^{(25,26)}$.

\section{CONCLUSIONS}

1- Early loss of primary incisors due to caries would have minor effect on speech development.

2- Replacement of prematurely lost primary incisors with fixed prosthetic appliances did not affect speech development.

3- Replacement of prematurely lost primary incisors with fixed prosthetic appliances to for the purpose of restoring appearance if required by the parents should be encouraged however, assuring that speech development will not be affected by early loss of these teeth should also be explained to them if they are concerned. 


\section{LIMITATION OF THE STUDY}

Variation in number of prematurely lost primary incisors and its effect on speech was not investigated in this study.

\section{REFERENCES}

1- Rodrigues de Sant AG, Rodrigues CRMD, Guedes-Pinto AC. Primary anterior tooth replacement with fixed prosthesis using precision connection system: A case report. Quintessence Int 2002; 33: 303-08.

2- Holan \& Needleman . Premature loss of primary anterior teeth due to trauma ? Potential short- and long-term sequelae. Dental Traumatology 2014; 30: 100-106; doi: 10.1111/edt.12081

3- Kessler HE. The relationship of dentistry to speech. J Amer Dent Assoc 1954;48:44-9.

4- Blyth P. The relationship between speech, tongue behaviour, and occlusal abnormalities. Dent Pract 1959;10:11-20.

5- Snow K. Articulation proficiency in relation to certain dental abnormalities. J Speech Hear Disord 1961;26:209-12.

6- Laine T. Associations between articulatory disorders in speech and occlusal anomalies. Eur J Orthod 1987;9:144-50.

7- Lamberghini F, Kaste LM, Fadavi S, Koerber A, Punwani IC, Smith EB. An association of premature loss of primary maxillary incisors with speech production of bilingual children. Pediatr Dent. 2012 Jul-Aug;34(4):307-11 .

8- Riekman GA, ElBadrawy HE. Effect of premature loss of primary maxillary incisors on speech.Pediatr Dent 1985;7:119-22.

9- Klapper BJ, Strizak-Sherwin R. Esthetic anterior space maintenance. Pediatr Dent 1983; 5(2): 121-23.

10- Waggoner WF, Kupietzky A. Anterior esthetic fixed appliances for the preschooler: considerations and a technique for placement. Pediatr Dent 2001;23(2):147-50.

11- Turgut M.D, Genc G.A, Basar F, Tekcicek M.U- The effect of early loss of anterior primary tooth on speech production in preschool children. Turk J. Med Sci 2012; 42(5): 867-875.

12- Steffen MJ, Miller JB, Johnson R. An esthetic method of anterior space maintenance. J Dent Child 1971; May-June: 154-57.

13- Jasmin JR, Groper JN. Fabrication of a more durable fixed anterior esthetic appliance. J Dent Child 1984; 2: 124-27

14- Margolis FS. The esthetic space maintainer. Compendium 2001; 22(11): 911-14
15- Kapur A, Chawla HS, Goyal A, Gauba K. An esthetic point of view in very young children. . J Clin Pediatr Dent 2005; 30(2): 99-104.

16- Patil RB, Rachappa MM. A Simple Modification of Fixed Space Maintainers for Replacement of An Avulsed maxillary Primary Central Incisor. International Journal of Dental Clinics 2011; 3(1): 117

17- Coston GN, Salinas CF. Speech characteristics in patients with hypohidrotic ectodermal dysplasia in Birth defects: Original article series 1988: 24:229-234.

18- Kotby MN, Bassiouny S, Elzomor M, Mohsen N: Pilot study for standardization of an articulation test. Proc 10th Annu Ain-Shams Med Congr, Cairo, 1985.

19- Krause JC1, Braida LD. Acoustic properties of naturally produced clear speech at normal speaking rates. J Acoust Soc Am. 2004 Jan;115(1):362-78.

20- Shariq M. Arabic and English Consonants: A Phonetic and Phonological Investigation Advances in Language and Literary Studies, 2015; 6 (6) ISSN: 2203-4714

21- Molly , L.; Nackaerts ,O.; Vandewiele, K.; Manders, E.; Steenberghe, D. and Jacobs, R., Speech adaptation after treatment of full edentulism through immediate - loaded implant protocols. Clin. Oral Lmpl. Res. 2008; 19: 86 - 90.

22- Zheng Shu-zhen. Comparison of the influence between the middle palatal bar denture and hole-rotating connector denture upon speech function. Chinese Journal of practical stomatology, 2010; $5: 20$.

23- Niemi M, Laaksonen JP, Pellomaki T, Kurimo J, Aallonen $\mathrm{O}$, and Happonen RP. Acoustic comparison of vowel sounds produced before and after orthognathic surgery for mandibular advancement. Journal Oral Maxillofacial Surgery 2006; $64: 910-916$

24- Gable TO, Kummer AW, Lee L, Creaghead NA, Moore LJ. Premature loss of the maxillary primary incisors: effect on speech production. ASDC J Dent Child. 1995 May-Jun; 62(3):173-9.

25-Koroluk LD, Riekman GA. Parental perceptions of the effects of maxillary incisor extractions in children with nursing caries. J Dent Child 1991:58:233-236,

26- Sowjanya V, Thakur MS, Prasad MG, Sahana S, Vasa AAK. Aesthetic Rehabilitation of a child with missing anterior teeth and Thumbsucking Habit using Fixed Functional Space maintainer in combination with Habit breaking Appliance. Annals and Essences of Dentistry 2013: 5 (1): 18-20. 